\title{
Reversing anticoagulants
}

Drugs that prevent blood coagulation are used during surgery or to treat acute thrombotic events, and prophylactically in individuals at risk of thrombosis. However, as these compounds are associated with an increased risk of bleeding, antidotes may be needed to reverse their effects. Two recently published papers describe such antidotes.

Unfractionated heparin (UFH), low-molecular-weight heparins (LMWHs) and the synthetic heparin pentasaccharide known as fondaparinux are the most commonly used anticoagulants in acute settings. Protamine sulphate, a cationic peptide that binds to heparins, is the only approved antidote, but it has some adverse effects (including allergic reactions) and is not completely effective against LMWHs and fondaparinux.

Shenoi et al. reasoned that a universal heparin reversal agent (UHRA) that would be effective against UFH, LMWHs and fondaparinux might be generated if the size of the molecule and the density of heparin-binding groups were optimized. They used a dendritic polymer scaffold that was based on hyperbranched polyglycerol, to which they attached trivalent cationic heparin-binding groups. Eight molecules were generated with different molecular weights and heparinbinding group stoichiometry, and one of them, UHRA-7, neutralized UFH and LMWHs in plasma and fondaparinux in whole-blood samples. In rats, UHRA-7 completely and rapidly neutralized LMWHs and UFH. UHRA-7 also stopped the bleeding associated with all the heparins and heparin-based anticoagulants in a mouse tail transection model.

UHRA-7 was safe and biocompatible - blood coagulation and complement activation were unaffected by the drug, and mice treated with UHRA-7 showed no signs of acute toxicity during the 29-day follow-up period. In addition, the fully synthetic nature of UHRA-7 would enable a low production cost.

There is no currently approved antidote for novel oral anticoagulants (NOACs; dabigatran, rivaroxaban, apixaban and edoxaban) that are now in widespread use as alternatives to warfarin for the long-term treatment of patients at risk of thrombosis. A second study published in The New England Journal of Medicine examined the effects of PER977, a small, synthetic, cationic molecule that was originally designed to bind to heparin but that also counteracts approved NOACs in vitro and in rat models. In a trial of 80 healthy individuals, a single intravenous dose of PER977, given 3 hours after edoxaban, restored blood clotting time to less than $10 \%$ above baseline within 10 minutes of administration. The effects lasted for at least 24 hours.

UHRA-7 has the potential to be used clinically in situations that require reversal of acute anticoagulation. PER977 could be useful in these situations too; however, as PER977 additionally reverses the NOACs, it could also be used in patients who are taking a NOAC and have unexpected bleeding. An agent that can reverse all available NOACs would be particularly important in situations where an antidote is urgently needed, before a patient's full history is known. Additional preclinical studies of UHRA-7 are ongoing; PER977 is in Phase II trials of patients receiving chronic edoxaban therapy.

Megan Cully

ORIGINAL RESEARCH PAPERS Shenoi, R. A. et al. Affinity-based design of a synthetic universal reversal agent for heparin anticoagulants. Sci. Transl Med. 6, 260ra150 (2014) | Ansell, J. E. et al. Use of PER977 to reverse the anticoagulant effect of edoxaban. N. Engl.J.Med. 371, 2141-2142 (2014) 